\title{
Decoration of buried surfaces in Si detected by positron annihilation spectroscopy
}

\author{
R. S. Brusa, ${ }^{\text {a) }}$ C. Macchi, S. Mariazzi, and G. P. Karwasz \\ Dipartimento di Fisica, Università di Trento, Via Sommarive 14, I-38050 Povo, Trento, Italy \\ W. Egger, P. Sperr, and G. Kögel \\ Institut für Angewandte Physik und Messtechnik, Universität der Bundeswehr München, \\ Werner-Heisenberg Weg 39, D-85577 Neubiberg, Germany
}

(Received 24 May 2005; accepted 15 November 2005; published online 6 January 2006)

\begin{abstract}
The terminations of buried surfaces of two different cavity types (nano- and microcavities) produced in the same $\mathrm{He}^{+}-\mathrm{H}^{+}$co-implanted $p$-type $\mathrm{Si}$ (100) sample annealed at $900{ }^{\circ} \mathrm{C}$, are studied and characterized by positron annihilation spectroscopy. The characterization was carried out by means of three complementary positron techniques: Doppler broadening and coincidence-Doppler broadening spectroscopy with a continuous slow positron beam, and lifetime spectroscopy with a pulsed slow positron beam. It was found that the nanocavities have a pristine surface of $\mathrm{Si}$, while the surfaces of the microcavities, formed below protruding blisters, are oxygen decorated. This case study opens the interesting use of the positron spectroscopy tool in the topical subject of empty space for microelectronics applications. (c) 2006 American Institute of Physics.
\end{abstract}

[DOI: $10.1063 / 1.2162691]$

Empty nano- and microvoids in silicon, organosilicates, carbon, and organic-polymer based materials are of wide technological interest for applications in microelectronics industry. There is a strong demand of insulators with nanoporosities and low dielectric constant to substitute $\mathrm{SiO}_{2}$ in new devices. ${ }^{1,2}$ A new technology has been developed to obtain empty spaces under Si layers, starting from deep trenches annealed in hydrogen atmosphere. ${ }^{3}$ Gate metal oxide semiconductor field-effect transistors have been realized with empty space in Si obtained by removing SiGe sacrificial layers. ${ }^{4}$ Nanocavities formed in Si by $\mathrm{H}$ and $\mathrm{He}$ implantation and subsequent annealing are used for efficiently gettering transition metals, ${ }^{5,6}$ while $\mathrm{H}$ and $\mathrm{He}$ co-implantation have a synergetic effect in forming blisters suspended on empty cavities $^{7}$ and can be used in the synthesis of silicon-oninsulator films by the ion-cut process. ${ }^{8}$

The knowledge of the bond terminations of the produced internal surfaces is essential in all the aforementioned applications. Decorations of buried surfaces, produced by chemical species present in the gas ambient during thermal treatments or by impurities (such as oxygen) present in the starting material, can occur. These decorations, changing the morphology of the surfaces, influence the electrical and gettering properties. Furthermore, it is of interest to obtain, with a nondestructive technique, a quantitative evaluation of the in-depth position of the micro- and nanocavities and vacancy clusters produced by ion implantation., ${ }^{9,10}$

The aim of this letter is to show that, with the positron annihilation spectroscopy (PAS), it is possible to gain information on the surface decoration of buried cavities of different types produced in the same sample. The studied system is p-type (100) Si (1.7-2.5 $\Omega \mathrm{cm}$ ), Czochralski-grown (about $6.6 \times 10^{17} \mathrm{~cm}^{-3}$ oxygen content), co-implanted at room temperature with $\mathrm{He}^{+}$ions at $30 \mathrm{keV}$ with a dose of 1 $\times 10^{16}$ ions $/ \mathrm{cm}^{2}$ and successively with $\mathrm{H}^{+}$ions at $24 \mathrm{keV}$

\footnotetext{
${ }^{a)}$ Electronic mail: brusa@science.unitn.it
}

with a dose of $1 \times 10^{16}$ ions $/ \mathrm{cm}^{2}$. The density of the beam current was about $8 \mu \mathrm{A} / \mathrm{cm}^{2}$ and the sample holder tilted by $7^{\circ}$ to reduce channeling effects. The analyzed sample was annealed in vacuum for $2 \mathrm{~h}$ at $900{ }^{\circ} \mathrm{C}$. At this annealing temperature $\mathrm{He}$ and $\mathrm{H}$ are found to be completely desorbed and empty cavities appear in the material. ${ }^{11}$ The $\mathrm{He}^{+}$and $\mathrm{H}^{+}$ co-implantation has an increasing applied interest because it was shown that co-implantation, in comparison to single $\mathrm{H}^{+}$ implantation, reduces the overall dose necessary to obtain $\mathrm{Si}$ exfoliation. ${ }^{12}$ The structure of the cavities in samples prepared in the same setup as described above, and annealed at 800 and $900{ }^{\circ} \mathrm{C}$ have been investigated by transmission electron microscopy (TEM). ${ }^{7}$ The draft in Fig. 1 presents the different defects detected by TEM after sample annealing at $900{ }^{\circ} \mathrm{C}$ : blisters of different shapes, above buried empty microcavities, spaced by a defective layer of distributed nanocavities and dislocation loops centered at a depth of about $290 \mathrm{~nm}$. Buried microcavities under the blisters can have an elongated shape with length up to $4 \mu \mathrm{m}$ and hundreds of nanometers wide, or a more irregular shape. The silicon layers above the buried microcavity have different thicknesses (in Ref. 7 a blister with a layer of $150 \mathrm{~nm}$ is characterized) and are plastically deformed.

Three PAS techniques ${ }^{13}$ have been employed to completely characterize the open volume structure of the sample and to obtain information on the surface terminations of the cavities. (1) Doppler broadening spectroscopy (DBS) in coincidence: to study the high-momentum region of the $511 \mathrm{keV}$ annihilation line, due to positron annihilation with core electrons. Annihilations with core electrons are a fingerprint of chemical elements and can give information on the

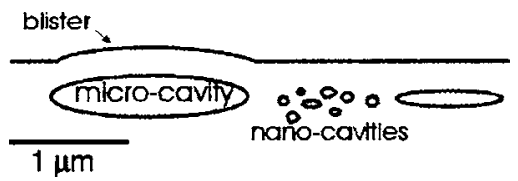

FIG. 1. Sketch of the defects morphology as detected by TEM (see Ref. 7). 


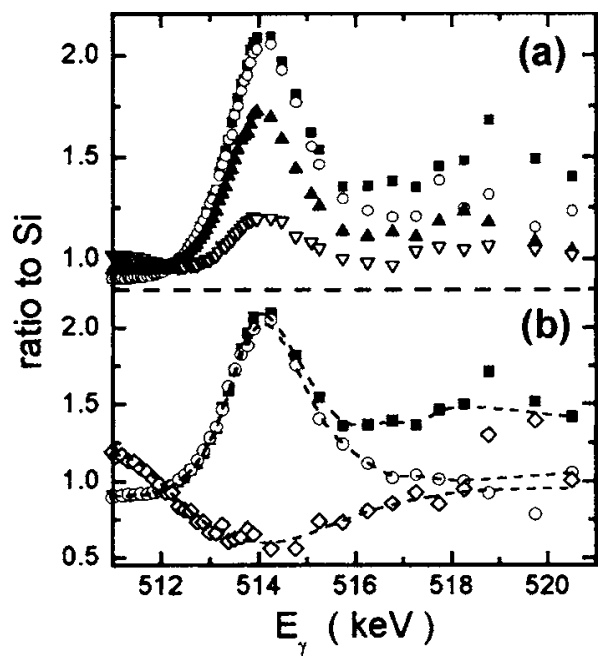

FIG. 2. (a) Ratio of the coincidence Doppler broadening spectrum, measured at different positron implantation energies $E$, to spectrum of bulk Si. Full squares $(E=0.15 \mathrm{keV})$, open circles $(E=1 \mathrm{keV})$, full triangles $(E$ $=3 \mathrm{keV})$, inverted triangles $(E=6.5 \mathrm{keV})$. (b) Characteristic ratio curves for positron annihilating at the surface (full squares), at the buried surfaces (open circles), in the clean nanocavities (open diamond), extracted from the measurements. The continuous lines are guides for the eyes.

decoration of open volume defects. ${ }^{14-16}$ (2) DBS: to study the low-momentum region around the $511 \mathrm{keV}$ peak position, due to positron annihilation with valence electrons. From DBS, the distribution of open volume defects and the positron trapping probability can be extracted. ${ }^{10,17}$ Both experiments were carried out with a continuous slow positron beam. ${ }^{18}$ Two high-purity germanium (HPGe) detectors $45 \%$ efficiency, $1.4 \mathrm{keV}$ resolution at $511 \mathrm{keV}$, in a $180^{\circ}$ configuration were used for coincidence-DBS while a HPGe (1.2 keV resolution) was used for DBS measurements. (3) Positron annihilation lifetime spectroscopy (PALS) with a pulsed slow positron beam with a total time resolution (pulsing plus detector system) of $230-250 \mathrm{ps},{ }^{19}$ to characterize unambiguously the type of open volume defects. Both positron beams used have a spot of some $\mathrm{mm}^{2}$ on the sample position and portions of the sample that contain many blisters are probed in each measurement.

The information about the surface terminations and chemical decorations is contained in the coincidence-DBS measurements of the $511 \mathrm{keV}$ annihilation line $C\left(E, E_{\gamma}\right)$ at a positron implantation energy $E . E_{\gamma}$ is the energy of the annihilation gamma ray. This information is highlighted by peaks or valleys in ratio curves constructed as the ratio between the $511 \mathrm{keV}$ annihilation line measured in the sample under investigation and a reference $511 \mathrm{keV}$ annihilation line $C_{b}\left(E_{\gamma}\right)$, measured in bulk $\mathrm{Si}$, that is, at high positron implantation energy. ${ }^{15,16}$ In Fig. 2(a) the ratio-curves obtained by measuring $C\left(E, E_{\gamma}\right)$ at four positron implantation energies $E$, are reported. Each curve $C\left(E, E_{\gamma}\right)$ is a linear combination of different terms: $\quad C\left(E, E_{\gamma}\right)=C_{S}\left(E_{\gamma}\right) f_{S}(E)+\Sigma_{j} C_{t, j}\left(E_{\gamma}\right) f_{t, j}(E)$ $+C_{b}\left(E_{\gamma}\right) f_{b}(E)$, where $C_{s}\left(E_{\gamma}\right)$ and $C_{t, j}\left(E_{\gamma}\right)$ are the characteristic annihilation line of positron annihilating at the sample surface and in the traps $j . f_{s}, f_{t, j}$, and $f_{b}$ are the fractions of positron annihilating at the sample surface, in the different traps $j$ and in the bulk state, respectively. The $C_{t, j}\left(E_{\gamma}\right) / C_{b}\left(E_{\gamma}\right)$ curves are related to the structure and decoration of the traps and can be obtained solving a system Downloaded 26 Feb 2007 to 200.5.106.13. Redistribution subject

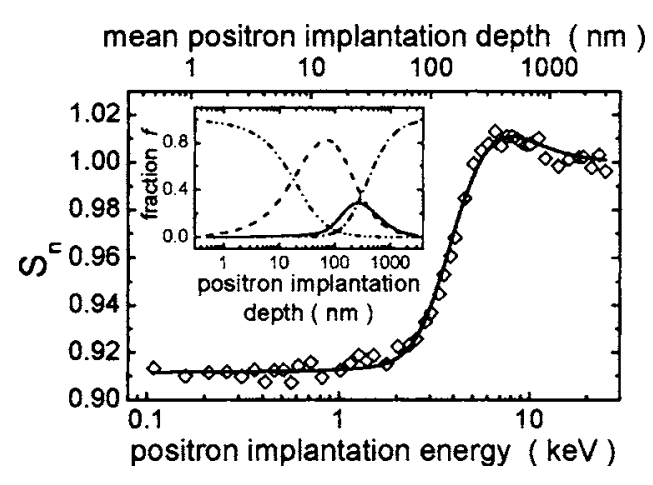

FIG. 3. $S_{n}$ parameter vs positron implantation energy $E$ (lower axis) and mean positron implantation depth (upper axis). The continuous line is the best fit to the data. Inset: positron annihilation probabilities vs $E: f_{s}$ at the surface (dash-dot-dot); $f_{t, 1}$ at the buried surface (dash); $f_{t, 2}$ in clean nanocavities (full line); $f_{b}$ in bulk (dash-dot).

formed with $j+1$ equations of the linear combination just described.

The positron fractions $\left(f_{s}, f_{t, j}, f_{b}\right)$, needed to solve the system are extracted by modeling the DBS measurements with the positron diffusion equation (details in Ref. 10). The Doppler broadening of the $511 \mathrm{keV}$ annihilation line is characterized by the shape parameter $S$. The $S$ parameter that characterizes the low-momentum region is defined as the ratio of the counts in the central area of the peak (our choice: $\left|511 \mathrm{keV}-E_{\gamma}\right| \leqslant 0.85 \mathrm{keV}$ ) and the total area of the peak $\left(\left|511 \mathrm{keV}-E_{\gamma}\right| \leqslant 4.25 \mathrm{keV}\right){ }^{13,17}$ The $S$ values were normalized to the Si bulk value $S_{b}\left(S_{n}=S / S_{b}\right)$. The $S_{n}$ values as a function of positron implantation energy $E$ are shown in Fig. 3. $E$ is related to the mean positron implantation depth $\bar{z}$ through the formula $\bar{z}=\left(40 E^{1.6}\right) / \rho$, with $\bar{z}$ in nm when the density $\rho$ and the $E$ are expressed in $\mathrm{g} / \mathrm{cm}^{3}$ and $\mathrm{keV}$, respectively. ${ }^{17}$ The best fit to the data of Fig. 3 was obtained with only two positron trap profiles: a derivative of a Gaussian, mean depth of $90 \pm 5 \mathrm{~nm}$, and a Gaussian centered at $290 \pm 5 \mathrm{~nm}(40 \pm 20 \mathrm{~nm}$ full width at half-maximum). The characteristic $S_{n}$ values of the two traps were found to be $S_{t, 1}=0.903$ and $S_{t, 2}=1.133$, respectively. The fractions $f$ are reported as a function of $E$ in the inset of Fig. 3. $f_{t, 1}$ and $f_{t, 2}$ are the fractions of positrons trapped in the two trap types. The positron effective diffusion length, which takes into account a possible surface field ${ }^{17}$ and dopant concentration, ${ }^{20}$ was found to be $100 \mathrm{~nm}$ by fitting $\operatorname{Si}(100)$ nonimplanted samples. The correctness of $L_{+}$used for fitting the damaged sample is confirmed a posteriori by the good agreement between the cavity depth distribution found by positron and the direct TEM observation. We have also tested that, due to the high trapping rate of the defects, variations up to more than $+50 \%$ of $L_{+}$change by less than $5 \%$ the values of the $f$, and this change does not affect the extracted $C_{s}\left(E_{\gamma}\right), C_{t, j}\left(E_{\gamma}\right){ }^{21}$

The $C_{s}\left(E_{\gamma}\right) / C_{b}\left(E_{\gamma}\right), C_{t, 1}\left(E_{\gamma}\right) / C_{b}\left(E_{\gamma}\right), C_{t, 2}\left(E_{\gamma}\right) / C_{b}\left(E_{\gamma}\right)$ curves, extracted solving the system, are now shown in Fig. 2(b). A variable average smoothing was applied to the ratio curves from $E_{\gamma}=515 \mathrm{keV}$. The spectra have been normalized to unity. The $C_{s}\left(E_{\gamma}\right) / C_{b}\left(E_{\gamma}\right)$ curve is due to positron annihilation in the native $\mathrm{SiO}_{2}$ at the $\mathrm{Si}$ external surface. The peak at $E_{\gamma}=514 \mathrm{keV}$ due to annihilation with oxygen atoms is well visible. ${ }^{16,22}$ The $C_{t, 2}\left(E_{\gamma}\right) / C_{b}\left(E_{\gamma}\right)$ is characteristic for annihilation in the traps with the more deep distribution: the high $S_{t, 2}$ value ${ }^{10,17}$ is a clear indication of annihilation in the nanocavities and the shape of the ratio curve shows that the
olP license or copyright, see http://apl.aip.org/apl/copyright.jsp 


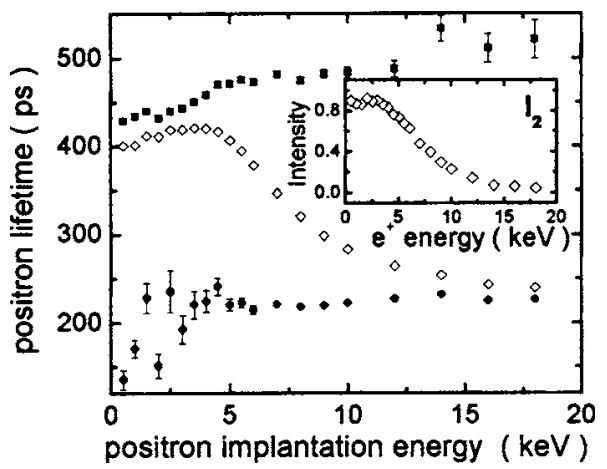

FIG. 4. Positron lifetimes as a function of positron implantation energy: full circles, $\tau_{1}$; full squares, $\tau_{2}$; open diamonds, mean lifetime. Inset: lifetime intensity $I_{2}$ vs $E$.

surfaces of these cavities have no decoration. ${ }^{16,21,23}$ The $C_{t, 1}\left(E_{\gamma}\right) / C_{b}\left(E_{\gamma}\right)$ curve is characteristic for the traps with the lower $S_{n}$; its peak is perfectly superimposed to that due to positron annihilation with oxygen atoms. These second traps are the surfaces of the microcavities below the blisters that turn out to be decorated by oxygen. The distribution (mean depth $90 \mathrm{~nm}$, asymmetric full width at half-maximum from 25 to $240 \mathrm{~nm}$ ), in this case, must be interpreted as the distribution of the mean thickness of the Si layer above the microcavities.

The analysis of the lifetime spectra, excluding the presence of other traps except micro- and nanocavities, strongly supports these results. The lifetime spectra, at each positron implantation energy, are perfectly fitted ${ }^{24}$ by two lifetimes $\tau_{1}$, and $\tau_{2}$ with intensities $I_{1}$ and $I_{2}$, respectively (see Fig. 4). The shorter lifetime, $\tau_{1}$, is the positron bulk lifetime in $\mathrm{Si}$ (225 ps). At low implantation energies, $\tau_{1}$ is reduced (about $150 \mathrm{ps}$ ) by the Si surface, which acts as a sink for freely diffusing positrons. In fact, at these low implantation energies the positron implantation depth $\langle z\rangle$ is $\leqslant 50 \mathrm{~nm}$, less than the diffusion length of $100 \mathrm{~nm}$, and the majority of positrons that are implanted in the first $100 \mathrm{~nm}$ below the surface, have a higher probability of reaching the surface in a minor time. From $5 \mathrm{keV}$ up, $\langle z\rangle$ is $200 \mathrm{~nm}$ up, and the implantation profile extends to more than $400 \mathrm{~nm}$ : about $60 \%$ of positrons are trapped with lifetime $\tau_{2}$, (see subsequent discussion), and only a small fraction of remaining positrons in the bulk state is at a distance less than $100 \mathrm{~nm}$ from the surface of the nano- and microdefects. The possible shortening of $\tau_{1}$, in this case, is too small to be observed. The longer lifetime $\tau_{2}$ that arises from positron annihilation at the $\mathrm{Si}$ surface and in the two traps, starts from about $420 \mathrm{ps}$ at low $E$ and reaches $480 \mathrm{ps}$ at $E=4.5 \mathrm{keV}$ (the variation of $I_{2}$ is shown in the inset of Fig. 4). The lifetime $\tau_{2}$ does not saturate, around $5 \mathrm{keV}$, at the positron lifetime in clean silicon nanocavities $(500-520 \mathrm{ps}) .{ }^{13,21}$ Indeed, the positron lifetime at the buried surfaces is expected to be similar to the lifetime of a positron trapped in the native $\mathrm{SiO}_{2}$ surface covering the $\mathrm{Si}$ sample (about $400 \mathrm{ps}$ ). The value of $480 \mathrm{ps}$ comes out as a combination between the lifetime of positron annihilating at the buried decorated surfaces and in the clean cavities. The lack of lifetimes shorter than $400 \mathrm{ps}$ and higher than $225 \mathrm{ps}$, in- dicates that the Si layer above micro- and nanocavities is free of vacancies or small vacancy clusters ${ }^{13}$ and confirms that the $S_{t, 1}$ value and the curve $C_{t, 1}\left(E_{\gamma}\right) / C_{b}\left(E_{\gamma}\right)$ are effectively correlated and characterize the surface below the blisters.

It is worth noting that the $S_{t, 1}(0.903)$ value characterizing the positron annihilation at the buried surfaces is near to $S$ values found in defected thermally grown $\mathrm{SiO}_{2} \cdot{ }^{17}$

Basic studies will be necessary to understand the process that brings oxygen to be present on the surfaces of the large cavities below blisters and not on the surface of the nanocavities.

This work was partially supported by the Ministero dell'Università e della Ricerca Scientifica e Tecnologica (MURST). The authors thank R. Tonini and G. Ottaviani for useful discussions.

${ }^{1}$ M. P. Petkov, C. L. Wang, M. H. Weber, K. G. Lynn, and K. P. Rodbell, J. Phys. Chem. B 107, 2725 (2003).

${ }^{2}$ R. S. Brusa, M. Spagolla, G. P. Karwasz, A. Zecca, G. Ottaviani, F. Corni, M. Bacchetta, and E. Carollo, J. Appl. Phys. 95, 2398 (2004).

${ }^{3}$ I. Mizushima, T. Sato, S. Taniguchi, and Y. Tsunashima, Appl. Phys. Lett. 77, 3290 (2000).

${ }^{4}$ J. Pretet, S. Monfray, S. Cristoloveanu, and T. Skotnicki, IEEE Trans. Electron Devices 51, 240 (2004).

${ }^{5}$ A. Paeva, P. F. P. Fichtner, D. L. da Silva, M. Behar, R. Koegler, and W. Skorupa, J. Appl. Phys. 91, 69 (2002).

${ }^{6}$ D. A. Brett, G. de M. Azevedo, D. J. Llewellyn, and M. C. Ridgway, Appl. Phys. Lett. 83, 946 (2003).

${ }^{7}$ S. Fabbroni, G. C. Gazzadi, L. Felisari, R. Tonini, F. Corni, and G. Ottavini, Appl. Phys. Lett. 85, 1683 (2004).

${ }^{8}$ C. H. Yun, N. Quitoriano, and N. W. Cheung, Appl. Phys. Lett. 82, 1544 (2003).

${ }^{9}$ V. C. Venezia, D. J. Eaglesham, T. E. Haynes, A. Agarwal, D. C. Jacobson, H. J. Gossmann, and F. H. Baumann, Appl. Phys. Lett. 73, 2980 (1998).

${ }^{10}$ R. S. Brusa, G. P. Karwasz, N. Tiengo, A. Zecca, F. Corni, R. Tonini, and G. Ottaviani, Phys. Rev. B 61, 10154 (2000).

${ }^{11}$ R. Tonini, F. Corni, C. Nobili, G. Ottaviani, F. Cazzaniga, and G. Queirolo, Solid State Phenom. 82-84, 291 (2002).

${ }^{12}$ A. Agarwal, T. E. Haynes, V. C. Venezia, O. W. Holland, and D. J. Eaglesham, Appl. Phys. Lett. 72, 1086 (1998).

${ }^{13}$ R. Krause-Rehberg and H. S. Leipner, Positron Annihilation in Semiconductors, Defect Studies, Springer Series in Solid State Science (Springer, Berlin, 1999).

${ }^{14}$ M. Alatalo, H. Kauppinen, K. Saarinen, M. J. Puska, J. Mäkinen, P. Hautojärvi, and R. M. Nieminen, Phys. Rev. B 51, 4176 (1995).

${ }^{15}$ P. Asoka-Kumar, M. Alatalo, V. J. Ghosh, A. C. Kruseman, B. Nielsen, and K. G. Lynn, Phys. Rev. Lett. 77, 2097 (1996).

${ }^{16}$ S. Szpala, P. Asoka-Kumar, B. Nielsen, J. P. Peng, S. Hayakawa, and K. G. Lynn, Phys. Rev. B 54, 4722 (1996).

${ }^{17}$ P. Asoka-Kumar, K. G. Lynn, and D. O. Welch, J. Appl. Phys. 76, 4935 (1994).

${ }^{18}$ A. Zecca, M. Bettonte, J. Paridaens, G. P. Karwasz, and R. S. Brusa, Meas. Sci. Technol. 9, 1 (1998).

${ }^{19}$ W. Bauer-Kugelmann, P. Sperr, G. Kögel, and W. Triftshäuser, Mater. Sci. Forum 363-365, 529 (2001).

${ }^{20}$ W. Bauer-Kugelmann, J. A. Duffy, J. Störmer, G. Kögel, and W. Triftshäuser, Appl. Surf. Sci. 116, 231 (1997).

${ }^{21}$ R. S. Brusa, C. Macchi, S. Mariazzi, G. P. Karwasz, W. Egger, P. Sperr, and G. Kögel, Phys. Rev. B 71, 245320 (2005).

${ }^{22}$ R. S. Brusa, W. Deng, G. P. Karwasz, A. Zecca, and D. Pliszka, Appl. Phys. Lett. 79, 149 (2001).

${ }^{23}$ M. Hakala, M. J. Puska, and R. M. Nieminen, Phys. Rev. B 57, 7621 (1998).

${ }^{24}$ Computer Program PATFIT, RISO National Laboratory, Denmark, 1998. 